\title{
UCP4C mediates uncoupled respiration in larvae of Drosophila melanogaster
}

\author{
Caterina Da-Ré ${ }^{1}$, Cristiano De Pittà ${ }^{1}$, Mauro A Zordan ${ }^{1}$, Giordano Teza ${ }^{2}$, Fabrizio Nestola², \\ Massimo Zeviani ${ }^{3}$, Rodolfo Costa ${ }^{1, *} \&$ Paolo Bernardi, ${ }^{4 *}$
}

\begin{abstract}
Larvae of Drosophila melanogaster reared at $23^{\circ} \mathrm{C}$ and switched to $14^{\circ} \mathrm{C}$ for $1 \mathrm{~h}$ are $0.5^{\circ} \mathrm{C}$ warmer than the surrounding medium. In keeping with dissipation of energy, respiration of Drosophila melanogaster larvae cannot be decreased by the F-ATPase inhibitor oligomycin or stimulated by protonophore. Silencing of Ucp $4 C$ conferred sensitivity of respiration to oligomycin and uncoupler, and prevented larva-to-adult progression at $15^{\circ} \mathrm{C}$ but not $23^{\circ} \mathrm{C}$. Uncoupled respiration of larval mitochondria required palmitate, was dependent on Ucp4C and was inhibited by guanosine diphosphate. UCP4C is required for development through the prepupal stages at low temperatures and may be an uncoupling protein.
\end{abstract}

Keywords Drosophila melanogaster; energy dissipation; mitochondria; respiration; uncoupling proteins

Subject Categories Metabolism

DOI 10.1002/embr.201337972 | Received 10 September 2013| Revised 11

February 2014 | Accepted 13 February 2014 | Published online 17 March 2014

EMBO Reports (2014) 15: 586-591

\section{Introduction}

The fruit fly Drosophila melanogaster originated in tropical Africa and colonized Europe only about 15,000 years ago (end of the last glaciation) eventually spreading to Australia and the Americas over the past few centuries [1]. This poikilotherm insect has become a cosmopolitan human commensal species, able to adapt to a wide range of environmental thermal conditions [2], yet the physiological bases for its climatic adaptations remain largely unknown. Moreover, while it is widely assumed that uncoupling proteins (UCPs) mediate mitochondrial thermogenesis in mammals, whether bona fide UCPs also exist in arthropods is the matter of debate $[3,4]$.

Nonshivering thermogenesis in mammals is mediated by UCP1, a $\mathrm{H}^{+}$-conducting protein that short-circuits the $\mathrm{H}^{+}$electrochemical gradient causing depolarization, stimulation of respiration and heat production [5]. UCP1 is selectively expressed in brown fat, the thermogenic tissue of newborn mammals and adult hibernating species [6,7], and in beige adipocytes after stimulation by cAMP $[8,9]$. UCP1 acts like a long-chain fatty acid- $\mathrm{H}^{+}$symporter [10], and indeed its activity strictly requires fatty acids that are released by PKA-dependent activation of hormone-sensitive lipase following adrenergic stimulation [7]. Homologs of UCP1 are expressed in mammals [11], plants [12], arthropods [13,14], C. elegans [15] and $X$. laevis [16]. Although a role as bona fide UCPs has not yet been demonstrated for any other UCP [6], it has been proposed that Arabidopsis UCP4 represents the ancestral UCP from which UCPs diverged [3]. Based on sequence homology, four UCPs have been identified in D. melanogaster, that is, UCP4A, 4B, 4C and 5 [3], and specific studies on Drosophila UCP5 have demonstrated that this species does not mediate uncoupling $[17,18]$.

Here, we have studied mitochondrial respiration in body wall preparations (BWPs) and isolated mitochondria from $D$. melanogaster instars II and III larvae after knockdown of Ucp $4 A, 4 B$ and $4 C$.

\section{Results and Discussion}

\section{Mitochondria of Drosophila larvae are uncoupled in situ}

Body wall preparations from III instar Drosophila larvae exhibited a steady oxygen consumption rate (OCR) of about $350 \mathrm{pmol} / \mathrm{min}$ that was not stimulated further by the addition of glucose, indicating that trehalose (provided with the medium) is sufficient to generate adequate glucose and respiratory substrates (Fig 1A). However, respiration was not inhibited by oligomycin, suggesting that in BWPs ATP is not produced by oxidative phosphorylation. In addition, OCR could not be stimulated by the uncoupler carbonylcyanide- $p$-trifluoromethoxyphenyl hydrazone (FCCP) even when the latter was added before oligomycin (Fig 1B). Respiration was largely inhibited by rotenone plus antimycin $\mathrm{A}$, demonstrating its mitochondrial origin (Fig $1 \mathrm{~A}$ and $\mathrm{B}$ ), and was inhibited by both iodoacetate (inhibitor of glyceraldehyde 3-phosphate dehydrogenase) and 2-deoxyglucose (which cannot be metabolized) indicating that it is fueled by

\footnotetext{
1 Department of Biology, University of Padova, Padova, Italy

2 Department of Geosciences, University of Padova, Padova, Italy

3 MRC Mitocondrial Biology Unit, University of Cambridge, Cambridge, UK

4 Department of Biomedical Sciences, Consiglio Nazionale delle Ricerche Institute of Neuroscience, University of Padova, Padova, Italy

*Corresponding author. Tel: +39 049827 6217; Fax: +39 049807 2213; E-mail: costa@bio.unipd.it

${ }^{\star *}$ Corresponding author. Tel: +39 049827 6365; Fax: +39 049827 6049; E-mail: bernardi@bio.unipd.it
} 


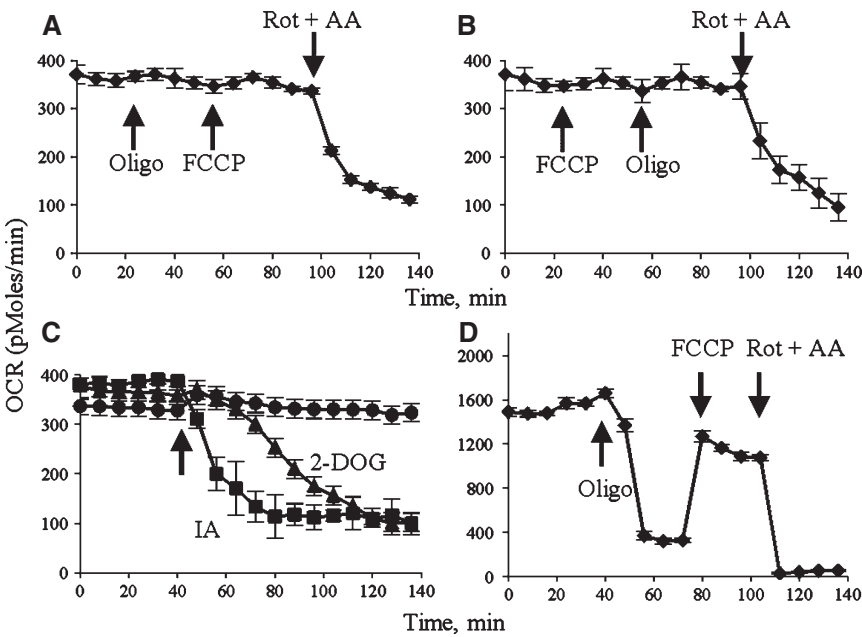

Figure 1. Oxygen consumption rate (OCR) of III instar larvae body wall preparations (BWPs) and of $\mathbf{S 2 R}^{+}$cells.

A, B Five micromolar oligomycin (Oligo), $1 \mu \mathrm{M}$ carbonylcyanide$p$-trifluoromethoxyphenyl hydrazone (FCCP) and $5 \mu \mathrm{M}$ rotenone plus $5 \mu \mathrm{M}$ antimycin $\mathrm{A}$ (Rot $+\mathrm{AA}$ ) were added to BWPs.

C Vehicle (circles), $20 \mathrm{mM}$ deoxyglucose (2-DOG, triangles) or $5 \mathrm{mM}$ iodoacetate (IA, squares) were added to BWPs.

D $5 \mu \mathrm{M}$ oligomycin, $1 \mu \mathrm{M}$ FCCP and $5 \mu \mathrm{M}$ rotenone plus $5 \mu \mathrm{M}$ antimycin $\mathrm{A}$ were added to $\mathrm{S} 2 \mathrm{R}^{+}$cells.

Data information: In all panels, additions are marked by arrows. In (A-C), data are mean \pm s.d. of 5 independent experiments with 20 BWPs each; in (D), data are mean \pm s.d. of 3 independent experiments with 20 cell incubations each.

glycolysis (Fig 1C). Not a single larva underwent metamorphosis, and all larvae died, when trehalose in the growth medium was replaced by pyruvate, lactate, galactose plus pyruvate, carnitine, octanoate, acetate, $\beta$-hydroxybutyrate or alanine, indicating that glycolysis is essential for development and survival. Based on these unexpected findings, we then asked whether they reflect a peculiar biological condition of mitochondria in early developmental stages of Drosophila. To answer this question, we performed OCR measurements in Drosophila Schneider 2 Receptor plus cells $\left(S 2 \mathrm{R}^{+}\right)$, which were derived from late embryonic stages and represent precursors to a variety of Drosophila tissues [19]. We found (as is usually seen with mammalian cells in culture) that respiration was inhibited by oligomycin and stimulated by FCCP (Fig 1D). Thus, lack of sensitivity to oligomycin and FCCP suggests that respiration of larvae is uncoupled.

\section{Larval mitochondria generate heat, while Ucp4C knockdown confers coupled respiration}

Genome analysis confirmed the existence of four putative Ucp genes in $D$. melanogaster that is, Ucp $4 A, U c p 4 B, U c p 4 C$ (which display a high homology with mammalian UCP4) [17] and Ucp5 (which displays a high homology with mammalian UCP5) [18]. Since it is already known that UCP5 does not mediate uncoupling in Drosophila $[17,18]$, we knocked down individual Ucp4 genes using the GAL4/ UAS binary yeast system $[20,21]$ and measured levels of silencing by qRT-PCR (Supplementary Fig S1) and respiratory features of
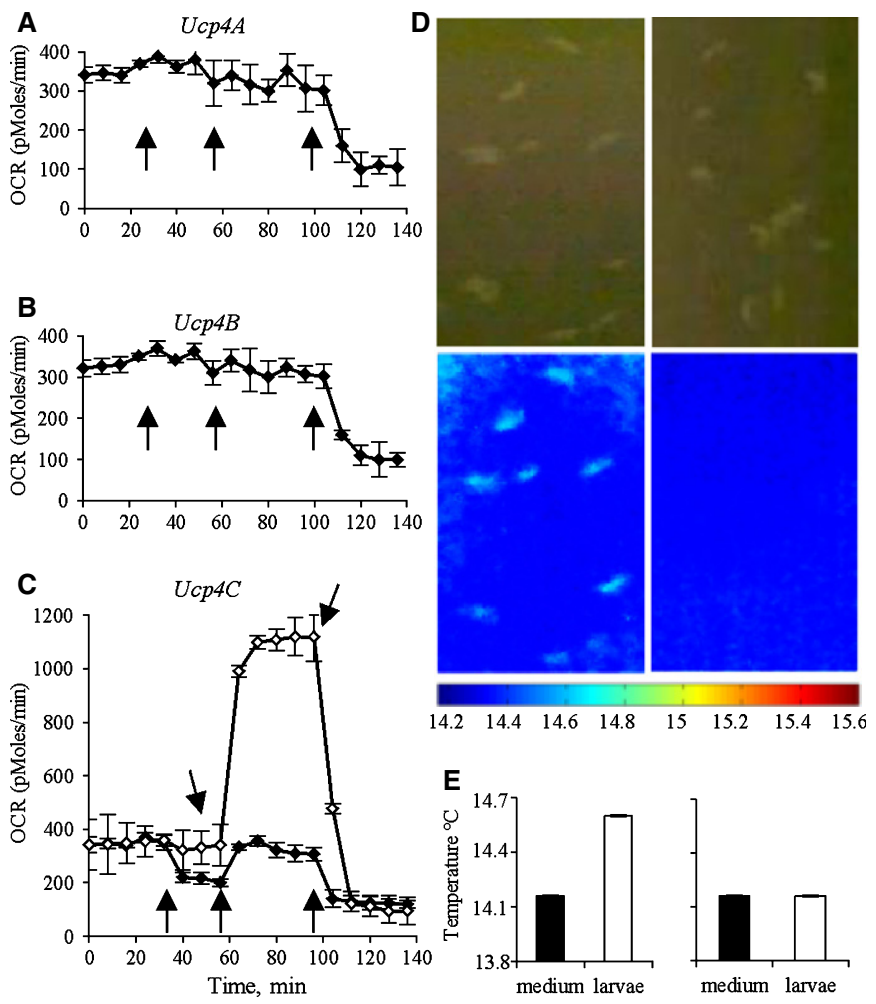

Figure 2. Effect of Ucp4 isoform silencing on oxygen consumption rate (OCR) of II instar body wall preparations (BWPs) and on heat production by III instar larvae.

UAS fly strains v6162 (UAS-Ucp4A), v33128 (UAS-Ucp4B) and v100064 (UAS-Ucp4C) were used.

A-C OCR of BWPs in Ucp4A- (A), Ucp4B- (B) or Ucp4C-interfered larvae (C). Where indicated (arrows) $5 \mu \mathrm{M}$ oligomycin, $1 \mu \mathrm{M}$ FCCP and $5 \mu \mathrm{M}$ rotenone plus $5 \mu \mathrm{M}$ antimycin A were added (closed symbols); open symbols (C) denote OCR upon the addition of FCCP at the first arrow, and of rotenone plus antimycin $\mathrm{A}$ at the second arrow.

D Visible light (upper panels) and infrared thermography images (lower panels) of wild-type (left panels) and Ucp4C-interfered larvae (right panels).

E Medium (filled bars) and larvae temperature (open bars) determined from the thermography measurements (average of 27 wild-type and 30 Ucp 4C-interfered larvae, error bar $=0.004^{\circ} \mathrm{C}$.

Data information: In $(\mathrm{A}-\mathrm{C})$, data are mean \pm s.d. of 5 independent experiments with 20 BWPs each.

BWPs derived from II instar larvae (Fig 2A-C). While downregulation of Ucp 4 transcripts did not affect the basal OCR (Fig 2A-C), only knockdown of $U c p 4 C$ made respiration sensitive to inhibition by oligomycin and to stimulation by FCCP (Fig 2C, closed symbols). Only in Ucp 4 C-interfered larvae addition of FCCP before oligomycin caused a threefold increase in respiration (Fig 2C, open symbols, compare with Supplementary Fig S2). The reason why FCCP is less effective when added after oligomycin in Ucp $4 C$-interfered larvae probably depends on ATP depletion and secondary $\mathrm{Ca}^{2+}$ deregulation affecting the mitochondrial permeability transition pore, an effect that is amplified in pore-related pathologies [22] and that can lead to respiratory inhibition due to depletion of matrix pyridine nucleotides $[23,24]$. This effect is obviously not seen when mitochondria are uncoupled (i.e. in wild-type and Ucp $4 A$ - and Ucp $4 B$ - 
interfered larvae) because in this case, ATP is not synthesized by mitochondria, and therefore, its levels are not affected by inhibition of F-ATP synthase by oligomycin. It should be noted that maximal respiration was higher in $U c p 4 C$-interfered than in wild-type larvae, possibly the result of increased mitochondrial biogenesis-an issue that will require further work. It should also be mentioned that inhibition of transcription was particularly effective for Ucp $4 C$ and $U c p 4 A$ but not for Ucp $4 B$ mRNAs (Supplementary Fig S1), yet neither UCP4A nor UCP4B can be responsible for uncoupling, or else interference of $U c p 4 C$ mRNA should not have had a phenotypic effect. We also tested a different set of knockdown Ucp4 lines with superimposable results (Supplementary Fig S3). To assess whether uncoupled respiration is linked to heat production, we used infrared thermography to study body temperature of wild-type and of Ucp $4 \mathrm{C}$ interfered larvae. Larvae were reared at $23^{\circ} \mathrm{C}$ and switched to $14^{\circ} \mathrm{C}$ for $1 \mathrm{~h}$, a protocol that does not affect development after larvae of either genotype are returned to $23^{\circ} \mathrm{C}$. The temperature of wild-type larvae was $0.5^{\circ} \mathrm{C}$ warmer than that of the surrounding medium, while no thermal gradient was maintained by Ucp $4 C$-interfered larvae (Fig 2D, E). Although we could not detect expression of Drosophila UCPs with antibodies against human UCP1, based on these results, it appears extremely likely that expression of Ucp $4 C$ mediates uncoupled respiration in Drosophila larvae and that UCP4C activity is associated with energy dissipation.

\section{UCP4C is essential for larval development at low temperatures}

To test whether expression of UCPs is essential for development, adult flies were mated and about 300 eggs (three replicates, each of about 100 eggs per genotype) from either wild-type or Ucp $4 A$-, $U c p 4 B$ - or $U c p 4 C$-interfered individuals were incubated at $23^{\circ} \mathrm{C}$ or $15^{\circ} \mathrm{C}$, that is, close to the minimal temperature allowing progression in development of wild-type $D$. melanogaster [25]. About the same number of larvae and adults was recorded at $23^{\circ} \mathrm{C}$ irrespective of genotype, while the number of III instar larvae was drastically reduced-and not a single adult developed-in Ucp $4 C$-interfered individuals at $15^{\circ} \mathrm{C}$ (Fig $3 \mathrm{~A}-\mathrm{C}$ ). The OCR of BWPs from III instar $U c p 4 A$ - and $U c p 4 B$-interfered larvae grown at $15^{\circ} \mathrm{C}$ displayed complete lack of response to oligomycin and FCCP (Fig 3D and E). Contrariwise, mitochondrial respiration was absent in Ucp $4 C$-interfered larvae (Fig 3F; notice that the residual OCR was nonmitochondrial, as it was insensitive to rotenone and antimycin A). This finding indicates a severe metabolic impairment, which is consistent with lack of further development.

\section{Uncoupled respiration of larval mitochondria requires palmitate and is inhibited by GDP}

Isolated mitochondria from III instar larvae were not constitutively uncoupled; in the absence of palmitate, they underwent the expected oligomycin-sensitive stimulation of respiration with ADP and uncoupling with FCCP (Fig 4, trace a'). Like in brown fat mitochondria [5-7], respiration could be stimulated by palmitate, inhibited by the pan-UCP inhibitor guanosine diphosphate (GDP) and stimulated by FCCP (Fig 4, trace a). These findings suggest that the uncoupling effect of palmitate depends on an endogenous UCP, which we propose to be $\mathrm{UCP} 4 \mathrm{C}$, for four reasons. First, $\mathrm{S}^{+} \mathrm{R}^{+}$cells that express only UCP4A (http://flyrnai.org) could not be stimulated by palmitate after permeabilization with digitonin (Fig 4, trace b) while they responded normally to ADP and FCCP (Fig 4, trace b'). Second, the uncoupling effect of palmitate was seen in wild-type mitochondria (Fig 4B) and after knockdown of Ucp4A (Fig 4C) or of $U c p 4 B$ (Fig 4D) while it was suppressed by knockdown of Ucp $4 C$ (Fig 4E); the effect of palmitate was insensitive to carboxyatractylate (Supplementary Fig S4), indicating that the adenine nucleotide translocator is not involved in the uncoupling effect [26]. Third, respiratory recoupling in BWPs was only observed after interference of Ucp 4C mRNA (Fig 2D). Fourth, among Drosophila Ucp isoforms, $U c p 4 C$ displays the highest homology to $U C P 1$, the gene encoding for the unique bona fide mammalian UCP1 [4,6] (Supplementary Fig S5).

It is apparent that uncoupled respiration in Drosophila requires a fatty acid, in keeping with results obtained in C. elegans $[15,27]$, Z. atratus [14] and G. cocquerelia [13] where palmitate was required to observe uncoupling. In Drosophila, the main source of fatty acids is triacylglycerol (TAG), which is hydrolyzed by activation of lipases, the most important being the Brummer TAG lipase, a homolog of the human adipocyte triglyceride lipase [28]. Larval fat body is composed essentially of TAG, while adult flies contain more phosphatidylglycerol [29]. TAG storage and mobilization are controlled by brain centers that regulate food intake and metabolism [30], suggesting that larvae respiratory activity in vivo is modulated by hormonal regulation of lipases. The details of the Drosophila signaling pathway(s) required to activate lipolysis as well as the fatty acid(s) responsible for uncoupling remain to be determined. We note that the endogenous species may be different from palmitate, and this could explain why respiration is not stimulated maximally by added palmitate. Of note, muscle-specific knockdown of Ucp $4 C$ had the same recoupling effect as ubiquitous silencing (Supplementary Fig S6), suggesting that muscle mitochondria are effectors of uncoupled respiration in Drosophila larvae.

\section{Physiological implications of UCP4C expression in D. melanogaster}

In temperate zones, D. melanogaster overwinters by entering an adult reproductive diapause characterized by a severe reduction in ovarian development $[25,31,32]$. The related physiological modifications allow adult flies to survive prolonged exposure to temperatures well below $12^{\circ} \mathrm{C}$ [32]. On the contrary, Drosophila larvae die in a few days at temperatures below $12^{\circ} \mathrm{C}[2,33]$ suggesting that larval stages represent a highly temperaturesensitive developmental window during Drosophila ontogeny. Moreover, larvae are way less mobile than adults and confined to their specific environmental niches. Uncoupled respiration could therefore have evolved as a transient adaptation favoring progression of larvae into more cold-tolerant developmental stages. It should be noted that UCP4C could also be required to allow fast turnover of the tricarboxylic acid cycle in order to provide biosynthetic intermediates required for growth, and/or to decrease production of reactive oxygen species, which is an exponential function of the mitochondrial membrane potential [34]. In summary, our data suggest that Drosophila metabolism during larval stages is fueled by glycolysis and uncoupled from ATP synthesis due to expression of UCP4C. 


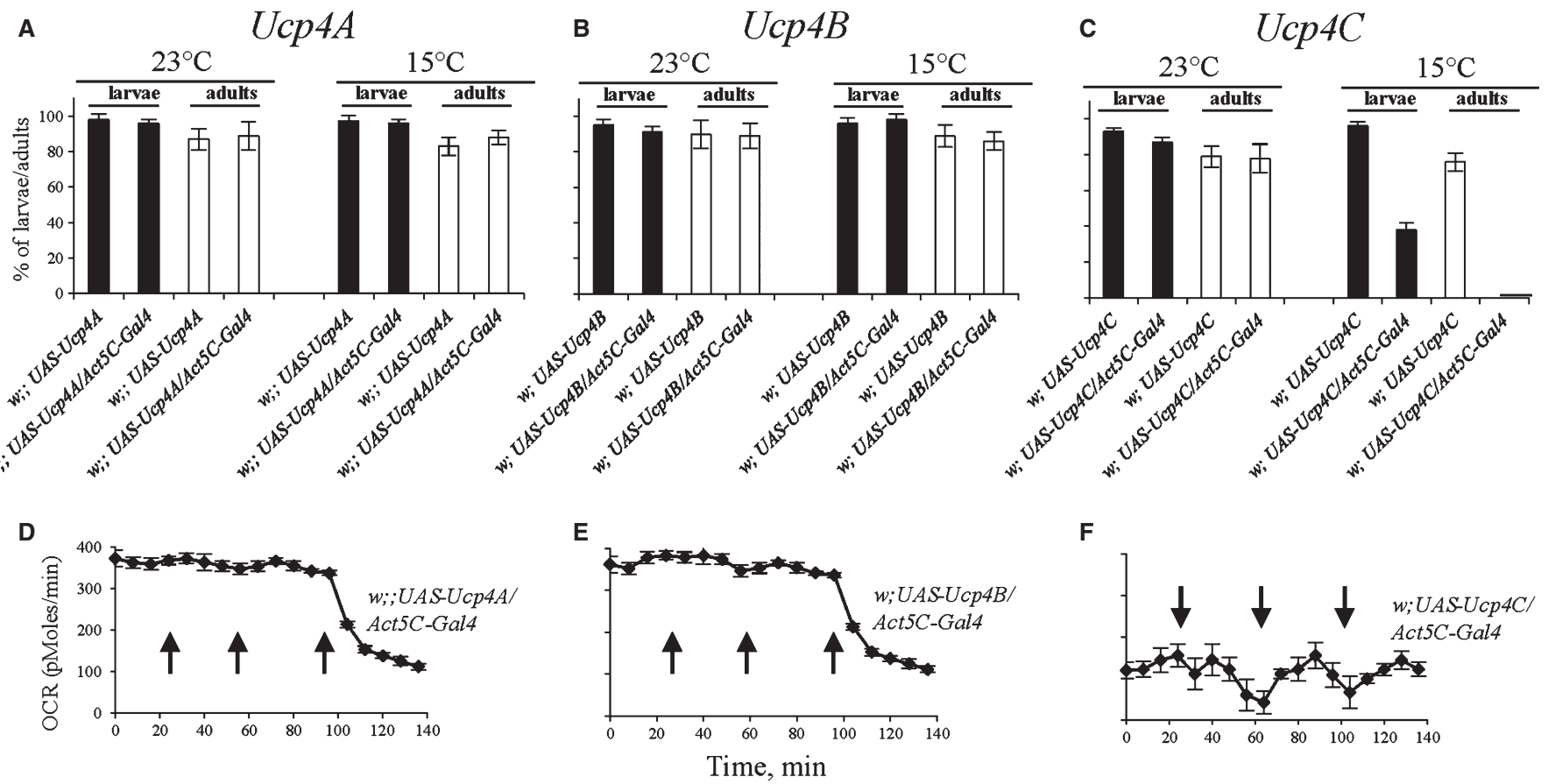

Figure 3. Effect of Ucp4 interference on egg-to-adult transition and oxygen consumption rate (OCR) of III instar larvae body wall preparations (BWPs).

A-C About 300 fertilized eggs (three replicates of $\approx 100$ each) were incubated at $23^{\circ} \mathrm{C}$ or $15^{\circ} \mathrm{C}$, and larvae (closed bars) and adults (open bars) were counted. $Y$-axis, $\%$ of larvae or adults developing from eggs of UAS- or UAS-Gal4-interfered flies.

D-F OCR of BWPs from Ucp4A- (D), UCp4B- (E) or Ucp4C-interfered larvae (F) grown at $15^{\circ} \mathrm{C}$. Where indicated (arrows) $5 \mu \mathrm{M}$ oligomycin, $1 \mu \mathrm{M}$ carbonylcyanidep-trifluoromethoxyphenyl hydrazone (FCCP) and $5 \mu \mathrm{M}$ rotenone plus $5 \mu \mathrm{M}$ antimycin A were added.

Data information: In (D-F), data are mean \pm s.d. of 3 independent experiments with 20 BWPs each.
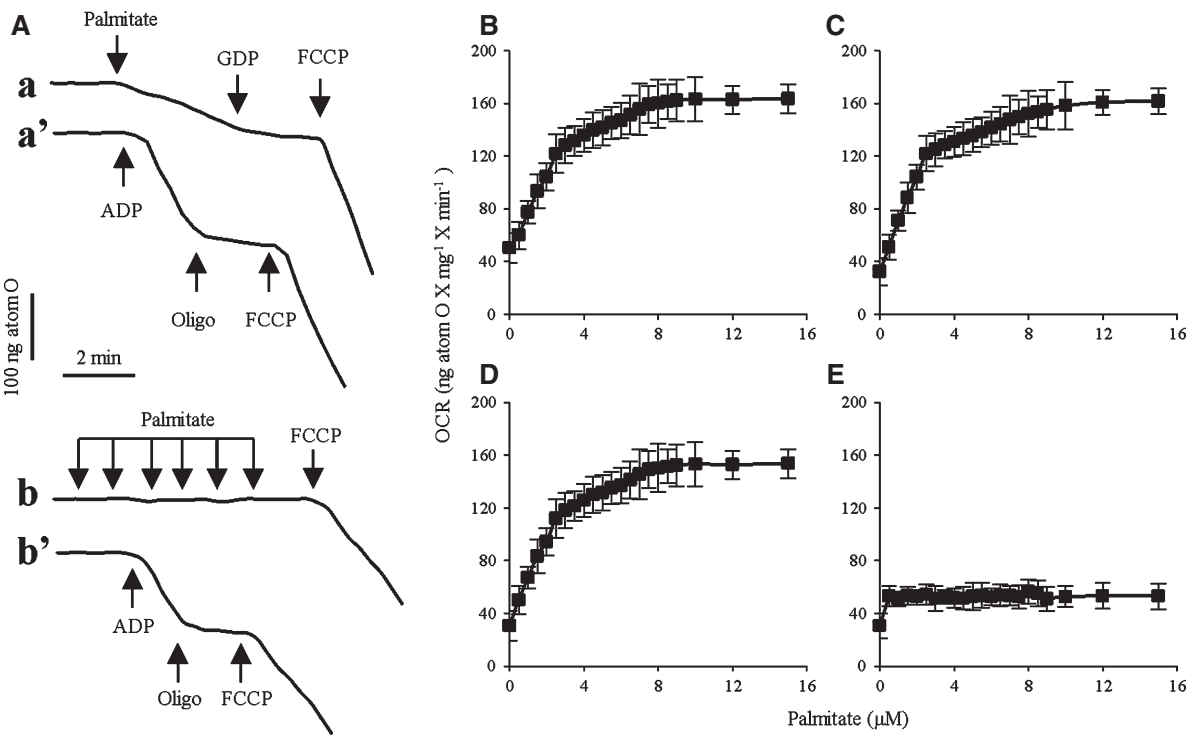

Figure 4. Effect of palmitate on oxygen consumption rates of larvae mitochondria and permeabilized $\mathbf{S}^{2} \mathbf{R}^{+}$cells.

A Mitochondria (2 mg) isolated from larvae (traces a, a') or $2 \times 10^{7} \mathrm{~S}^{2} \mathrm{R}^{+}$digitonin-permeabilized cells (traces b, b') were energized with $10 \mathrm{mM}$ succinate (final volume $2 \mathrm{ml}$ ). Where indicated by arrows additions were palmitate (15 $\mu \mathrm{M}$ in trace a and $5 \mu \mathrm{M}$ for each addition in trace b), $0.1 \mathrm{mM}$ ADP, $2 \mathrm{mM}$ GDP, $1 \mu \mathrm{g} / \mathrm{ml}$ oligomycin (Oligo) and $50 \mathrm{nM}$ carbonylcyanide- $p$-trifluoromethoxyphenyl hydrazone (FCCP). Results are representative of at least three independent experiments.

B-E Experiments were carried out on larval mitochondria isolated from wild-type (B) or from Ucp4A- (C), Ucp4B- (D) or Ucp4C-interfered larvae (E). OCR refers to the values measured after addition of the indicated concentrations of palmitate (mean from 3 to 5 determinations \pm s.d.). Maximal respiration (i.e. oxygen consumption after the addition of $50 \mathrm{nM} \mathrm{FCCP}$ ) was $746 \pm 16 \mathrm{ng}$ atom $\mathrm{O} \times$ per $\mathrm{mg} \times$ per $\min$ (mean \pm s.d. of all preparations used in B-D, $n=13$ ). 


\section{Materials and Methods}

\section{Fly strains}

UAS fly strains v6162, v102571, v33128, v33130, v100064 and v2647 were from the Vienna Drosophila RNAi Center (VDRC) [21]. The knockdown of Drosophila genes was achieved using the GAL4/ UAS system to target the RNAi [20]. Act5C-Gal4 and 24B-Gal4 drivers (Bloomington Stock Center) were used to activate interference of UCPs. All flies were raised on standard cornmeal medium and were maintained at $23^{\circ} \mathrm{C}(70 \%$ relative humidity) on a $12 \mathrm{~h}$ light : $12 \mathrm{~h}$ dark cycle.

\section{Body wall preparations and cell cultures}

A small portion of the tip was cut from larvae, internal organs were removed by gently squeezing from end to end, and the preparation was turned inside-out by rolling the cuticula along a holding tweezer. Each BWP was placed into one well of the Seahorse 24-well culture plate, held in place with a grid and covered with HL3A medium (115 mM sucrose, $70 \mathrm{mM} \mathrm{NaCl}, 20 \mathrm{mM} \mathrm{MgCl}_{2}, 10 \mathrm{mM} \mathrm{NaHCO}_{3}$, $5 \mathrm{mM} \mathrm{KCl}, 5 \mathrm{mM}$ HEPES, $5 \mathrm{mM}$ threalose). Twenty preparations were used in each experiment, and OCR was measured immediately. Drosophila S2R ${ }^{+}$cells were grown in Schneider's insect medium (Life Technologies) containing $10 \%$ heat-inactivated fetal bovine serum (Sigma-Aldrich).

\section{Oxygen consumption measurements}

Oxygen consumption rate was measured at $25^{\circ} \mathrm{C}$ either with a Seahorse XF24 Extracellular Flux Analyzer (Seahorse Bioscience, Billerica, MA) (BWPs and intact cells) or with a Clark-type oxygen electrode (isolated mitochondria and permeabilized cells). The XF24 Extracellular Flux Analyzer measures the OCR through a sensor cartridge embedded with a fluorescent sensor for oxygen coupled to a fiber-optic waveguide. The waveguide delivers light at $532 \mathrm{~nm}$ and transmits a fluorescent signal through optical filters $(650 \mathrm{~nm}$ for oxygen) to highly sensitive photodetectors [35]. The biosensor is much smaller than the BWPs and detects respiration in the region immediately below it, making the measurements independent of BWP size. S2R ${ }^{+}$cells were seeded at 20,000 cells/well and cultured for $48 \mathrm{~h}$, the medium being replaced with serum-free Schneider's medium immediately before the measurements. Mitochondrial isolation from larvae and permeabilization of $\mathrm{S}_{2} \mathrm{R}^{+}$cells were performed as described [36,37]. Respiratory measurements on mitochondria or permeabilized cells were performed in a medium containing $130 \mathrm{mM} \mathrm{KCl}, 10 \mathrm{mM}$ MOPS-Tris, $5 \mathrm{mM}$ succinate-Tris, $10 \mu \mathrm{M}$ EGTA and $2 \mu \mathrm{M}$ rotenone (final $\mathrm{pH}$ 7.4). Oligomycin, FCCP, rotenone and antimycin A were obtained from Life Technologies, and optimal concentrations were determined by titrations. Respiratory rates are average \pm s.d. of at least 60 individual BWP per condition, or of three independent $\mathrm{S}_{2} \mathrm{R}^{+}$and larval mitochondrial preparations.

\section{RNA isolation and QRT-PCR experiments}

Total RNA was isolated from 10 larvae with Trizol (Life Technologies) and further purified with $8 \mathrm{M} \mathrm{LiCl}$. RNA samples were checked for integrity by capillary electrophoresis (RNA 6000 Nano LabChip, Agilent Technologies). One microgram of total RNA per sample was used for first-strand cDNA synthesis using oligo-dT and SuperScript II reverse transcriptase (Life Technologies), and PCR-amplified with SYBR Green chemistry (Promega). Gene-specific primers were designed using Roche Application Design Tool (Roche Applied Science), and qRT-PCR was performed in the Applied Biosystems SDS-7500 thermal cycler (Life Technologies). Primer sequences are reported in Supplementary Table S1. To calculate the relative expression ratio, we used the $\Delta \Delta \mathrm{Ct}$ method implemented in the software of the Applied Biosystems thermal cycler [38], and Rp49 was used as endogenous control. mRNA levels are expressed relative to the quantity of template in the sample (RQ).

\section{Infrared thermography}

Both wild-type and interfered larvae were reared at $23^{\circ} \mathrm{C}$ and switched to $14^{\circ} \mathrm{C}$ for $1 \mathrm{~h}$ before thermographic recording. The measurements were carried out with a FLIR T620 camera, which is characterized by a $480 \times 640$ pixel bolometric array (technical data available online at http://www.flirthermography.co.uk/cameras/ camera/1079/). The thermal images acquired by the camera were post-calibrated on the basis of the acquisition distance, mean infrared reflectance of the observed object, reflected temperature and air temperature by means of FLIR QuickReport freeware package (download page: http://www.flir.com/thermography/eurasia/ en/content/?id =11368). The images were subsequently exported as temperature matrices stored in ASCII files for processing and analysis in MATLAB.

Supplementary information for this article is available online:

http://embor.embopress.org

\section{Acknowledgements}

We would like to thank Barbara Cannon, Paul Crichton, Fabio Di Lisa, Judy Hirst, Edmund Kunji, Yang Lee and Jan Nedergaard for their helpful comments. This work was funded by grants from Fondazione Cariparo (Progetti di Eccellenza) and the University of Padova (Progetti Strategici di Ateneo) Models of Mitochondrial Diseases, Telethon Program Project Therapeutic Strategies to Combat Mitochondrial Disorders, and Telethon MitMed: A Multicenter Consortium for the Identification and Characterization of Nuclear Genes Responsible for Human Mitochondrial Disorders. The infrared camera was purchased within the SMILAND Research Project funded by Fondazione Cariparo.

\section{Author contributions}

PB, RC and CDR conceived the study; MAZ, MZ and FN provided critical advice and suggested experiments; CDR and GT performed experiments; CDP designed qRT-PCR experiments; CDR, CDP and GT performed statistical analyses; PB, CDR and RC wrote the manuscript.

\section{Conflict of interest}

The authors declare that they have no conflict of interest.

\section{References}

1. Stephan W, Li H (2007) The recent demographic and adaptive history of Drosophila melanogaster. Heredity (Edinb) 98: 65-68 
2. Hoffmann AA (2010) Physiological climatic limits in Drosophila: patterns and implications. J Exp Biol 213: 870-880

3. Hanak P, Jezek P (2001) Mitochondrial uncoupling proteins and phylogenesis-UCP4 as the ancestral uncoupling protein. FEBS Lett 495: 137-141

4. Sokolova IM, Sokolov EP (2005) Evolution of mitochondrial uncoupling proteins: novel invertebrate UCP homologues suggest early evolutionary divergence of the UCP family. FEBS Lett 579: 313-317

5. Nicholls DG (2006) The physiological regulation of uncoupling proteins. Biochim Biophys Acta 1757: 459-466

6. Ledesma A, de Lacoba MG, Rial E (2002) The mitochondrial uncoupling proteins. Genome Biol 3: 3015.1-3015.9.

7. Cannon B, Nedergaard J (2004) Brown adipose tissue: function and physiological significance. Physiol Reu 84: 277-359

8. Wu J, Bostrom P, Sparks LM, Ye L, Choi JH, Giang AH, Khandekar M, Virtanen KA, Nuutila P, Schaart G et al (2012) Beige adipocytes are a distinct type of thermogenic fat cell in mouse and human. Cell 150: $366-376$

9. Wu J, Cohen P, Spiegelman BM (2013) Adaptive thermogenesis in adipocytes: is beige the new brown? Genes Deu 27: 234-250

10. Fedorenko A, Lishko PV, Kirichok Y (2012) Mechanism of fatty-aciddependent UCP1 uncoupling in brown fat mitochondria. Cell 151: $400-413$

11. Ricquier D, Bouillaud $F(2000)$ The uncoupling protein homologues: UCP1, UCP2, UCP3, StUCP and AtUCP. Biochem J 345(Pt 2): 161-179

12. Laloi M, Klein M, Riesmeier JW, Muller-Rober B, Fleury C, Bouillaud F, Ricquier D (1997) A plant cold-induced uncoupling protein. Nature 389: 135-136

13. Slocinska M, Antos-Krzeminska N, Rosinski G, Jarmuszkiewicz W (2011) Identification and characterization of uncoupling protein 4 in fat body and muscle mitochondria from the cockroach Gromphadorhina cocquereliana. J Bioenerg Biomembr 43: 717-727

14. Slocinska M, Antos-Krzeminska N, Rosinski G, Jarmuszkiewicz W (2012) Molecular identification and functional characterisation of uncoupling protein 4 in larva and pupa fat body mitochondria from the beetle Zophobas atratus. Comp Biochem Physiol B Biochem Mol Biol 162: $126-133$

15. Ji C, Guo W, Zhang M, Lu X, Ni Y, Guo X (2012) Caenorhabditis elegans ucp-4 regulates fat metabolism: suppression of ucp-4 expression induced obese phenotype and caused impairment of insulin like pathway. Gene 491: 158-164

16. Keller PA, Lehr L, Giacobino JP, Charnay Y, Assimacopoulos-Jeannet F, Giovannini N (2005) Cloning, ontogenesis, and localization of an atypical uncoupling protein 4 in Xenopus laevis. Physiol Genomics 22: 339-345

17. Fridell YW, Sanchez-Blanco A, Silvia BA, Helfand SL (2004) Functional characterization of a Drosophila mitochondrial uncoupling protein. J Bioenerg Biomembr 36: 219-228

18. Sanchez-Blanco A, Fridell YW, Helfand SL (2006) Involvement of Drosophila uncoupling protein 5 in metabolism and aging. Genetics 172: $1699-1710$

19. Schneider I (1972) Cell lines derived from late embryonic stages of Drosophila melanogaster. J Embryol Exp Morphol 27: 353-365

20. Brand AH, Perrimon N (1993) Targeted gene expression as a means of altering cell fates and generating dominant phenotypes. Development 118: $401-415$

21. Dietzl G, Chen D, Schnorrer F, Su KC, Barinova Y, Fellner M, Gasser B, Kinsey K, Oppel S, Scheiblauer S et al (2007) A genome-wide transgenic
RNAi library for conditional gene inactivation in Drosophila. Nature 448: $151-156$

22. Angelin A, Bonaldo P, Bernardi P (2008) Altered threshold of the mitochondrial permeability transition pore in Ullrich congenital muscular dystrophy. Biochim Biophys Acta 1777: 893-896

23. Vinogradov A, Scarpa A, Chance B (1972) Calcium and pyridine nucleotide interaction in mitochondrial membranes. Arch Biochem Biophys 152: $646-654$

24. Di Lisa F, Menabò R, Canton M, Barile M, Bernardi P (2001) Opening of the mitochondrial permeability transition pore causes depletion of mitochondrial and cytosolic $\mathrm{NAD}^{+}$and is a causative event in the death of myocytes in postischemic reperfusion of the heart. J Biol Chem 276: $2571-2575$

25. Schiesari L, Kyriacou CP, Costa R (2011) The hormonal and circadian basis for insect photoperiodic timing. FEBS Lett 585: 1450-1460

26. Andreyev AY, Bondareva TO, Dedukhova VI, Mokhova EN, Skulachev VP, Tsofina LM, Volkov NI, Vygodina TV (1989) The ATP/ADP-antiporter is involved in the uncoupling effect of fatty acids on mitochondria. Eur J Biochem 182: $585-592$

27. Iser WB, Kim D, Bachman E, Wolkow C (2005) Examination of the requirement for ucp-4, a putative homolog of mammalian uncoupling proteins, for stress tolerance and longevity in C. elegans. Mech Ageing Dev 126: $1090-1096$

28. Grönke S, Mildner A, Fellert S, Tennagels N, Petry S, Müller G, Jäckle H, Kühnlein RP (2005) Brummer lipase is an evolutionary conserved fat storage regulator in Drosophila. Cell Metab 1: 323-330

29. Carvalho M, Sampaio JL, Palm W, Brankatschk M, Eaton S, Shevchenko A (2012) Effects of diet and development on the Drosophila lipidome. Mol Syst Biol 8: 600

30. Kaun KR, Heberlein U (2009) Too fat to fly? New brain circuits regulate obesity in Drosophila. Neuron 63: 279-281

31. Allen MJ (2007) What makes a fly enter diapause? Fly (Austin) 1: $307-310$

32. Saunders DS, Bertossa RC (2011) Deciphering time measurement: the role of circadian 'clock' genes and formal experimentation in insect photoperiodism. J Insect Physiol 57: 557-566

33. David J, Clavel MF (1967) Influence de la température subie au cours du développement sur divers caractères biométriques des adultes de Drosophila melanogaster Meigen. J Insect Physiol 13: 717-729

34. Skulachev VP (1996) Role of uncoupled and non-coupled oxidations in maintenance of safely low levels of oxygen and its one-electron reductants. Q Reu Biophys 29: 169-202

35. Wu M, Neilson A, Swift AL, Moran R, Tamagnine J, Parslow D, Armistead S, Lemire K, Orrell J, Teich J et al (2007) Multiparameter metabolic analysis reveals a close link between attenuated mitochondrial bioenergetic function and enhanced glycolysis dependency in human tumor cells. Am J Physiol Cell Physiol 292: C125-C136

36. Ghezzi D, Arzuffi P, Zordan M, Da Ré C, Lamperti C, Benna C, D’Adamo P, Diodato D, Costa R, Mariotti C et al (2011) Mutations in TTC19 cause mitochondrial complex III deficiency and neurological impairment in humans and flies. Nat Genet 43: 259-263

37. von Stockum S, Basso E, Petronilli V, Sabatelli P, Forte MA, Bernardi P (2011) Properties of $\mathrm{Ca}^{2+}$ transport in mitochondria of Drosophila melanogaster. J Biol Chem 286: $41163-41170$

38. Livak KJ, Schmittgen TD (2001) Analysis of relative gene expression data using real-time quantitative PCR and the $2-\Delta \Delta \mathrm{Ct}$ Method. Methods 25 : $402-408$ 